\title{
The management of compensatory sweating after thoracic sympathectomy
}

\author{
Hidehiro Yamamoto, MD, and Masayoshi Okada, MD
}

\section{ABSTRACT}

Objective: The main therapeutic method of treatment for local hyperhidrosis is endoscopic thoracic sympathectomy. Generally, resections of the sympathetic trunk or ganglia are performed between the second rib and sixth rib. However, this procedure can result in compensatory sweating, in which excess sweating occurs on the back, chest, and abdomen. Compensatory sweating has been regarded as a thermoregulatory response and thought to be untreatable. This study suggests that compensatory sweating is not a physiologic reaction and is indeed treatable.

Methods: Eight patients with severe compensatory sweating were treated by observing blood perfusion of the skin with laser speckle flowgraphy, which determines the sympathetic nerves related to the area of skin with compensatory sweating. When intraoperative monitoring with laser speckle flowgraphy indicated the position of compensatory sweating by electrical stimulation of the sympathetic ganglion, ganglionectomy was performed.

Results: The skin domain that each sympathetic nerve controls was able to be detected by laser speckle flowgraphy. In all patients, compensatory sweating was resolved after interruption of the ganglia or sympathetic nerves related to compensatory sweating.

Conclusions: Our results demonstrate that compensatory sweating is caused by denatured sympathetic nerves influenced by endoscopic thoracic sympathectomy and is not the result of a physiological response. With laser speckle flowgraphy, the sympathetic nerve related to the sweating of various parts of the body could be identified. The treatment of compensatory sweating on the back, chest, and stomach was previously considered to be difficult; however, compensatory sweating is demonstrated to be treatable with this technique. (J Thorac Cardiovasc Surg 2019;158:1481-8)

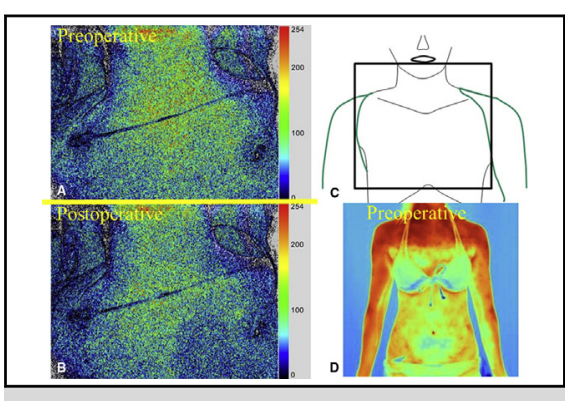

A decrease in the skin blood perfusion was observed by stimulating sympathetic nerve.

\section{Central Message}

Compensatory sweating is caused by denatured sympathetic nerves. Excision of the appropriate ganglia is an effective treatment for compensatory sweating.

\section{Perspective}

Compensatory sweating is caused by denatured sympathetic nerves influenced by thoracic sympathectomy. The sympathetic ganglia associated with compensatory sweating can be identified intraoperatively by observing blood perfusion of the skin using laser speckle flowg raphy. Excision of the appropriate ganglia is an effective treatment for compensatory sweating.

See Commentaries on pages 1489 and 1490.
Hyperhidrosis, which is characterized by excessive sweating of the face, axilla, and hands, is not a rare disease. Patients with palmar hyperhidrosis are estimated to represent $2.78 \%$ of the Japanese population and $3 \%$ of

From the Department of Surgery, Yamamoto-Hidehiro Clinic, Tokyo, Japan.

There were no sources of funding to declare.

Clinical trial registry number YHC20151208

Institutional review board approval CRB20151222-1 was obtained December 22, 2015.

Received for publication Oct 16, 2018; revisions received May 14, 2019; accepted for publication May 16, 2019; available ahead of print July 26, 2019.

Address for reprints: Hidehiro Yamamoto, MD, Department of Surgery, YamamotoHidehiro Clinic, 2-28-4 Dogenzaka, Shibuya-ku, Tokyo 150-0043, Japan (E-mail: bestets@yahoo.co.jp).

$0022-5223 / \$ 36.00$

Copyright (c) 2019 by The American Association for Thoracic Surgery

https://doi.org/10.1016/j.jtcvs.2019.05.062 the North American population. ${ }^{1,2}$ The main therapeutic intervention is endoscopic thoracic sympathectomy (ETS). More than 13,000 patients have undergone ETS at our clinic. Generally, resections of the sympathetic trunk or ganglia are performed at points between the second and sixth ribs. A side effect of this procedure, however, is compensatory sweating (CS), which is characterized by excessive sweating of the back, chest, and abdomen.,

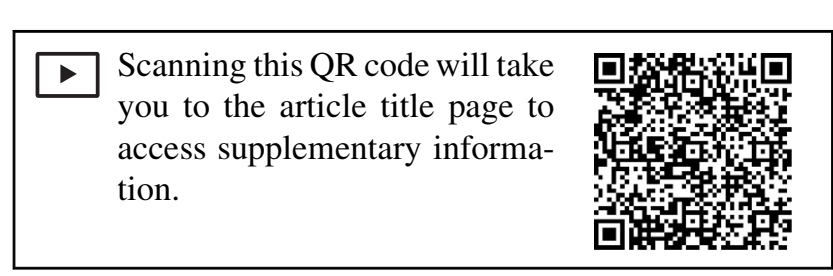




\section{Abbreviations and Acronyms}

ETS = endoscopic thoracic sympathectomy

$\mathrm{CS}=$ compensatory sweating

$\mathrm{LSFG}=$ laser speckle flowgraphy

CS has resulted in many medical lawsuits occurring in Japan and was formerly believed to be untreatable. CS was regarded as a thermoregulatory response resulting in anhidrosis of the head, neck, and upper extremities. ${ }^{3-6}$ Various methods to ameliorate CS have been suggested, including interruption of the sympathetic chain at fewer or lower levels, transection of only the rami while maintaining the sympathetic chain intact, and clipping of the sympathetic chain. None of these methods, however, appeared to have a demonstrable effect on the occurrence of CS. ${ }^{5}$ There is thus currently no evidence supporting a complete treatment option for CS. In the study presented here, we aimed to treat CS through identification of the sympathetic nerves responsible for CS.

\section{PATIENTS AND METHODS}

Between February 2016 and April 2017, a total of 8 patients with severe CS after sympathectomy were included in this study, which was approved by the institutional review board at Yamamoto-Hidehiro Clinic. Each
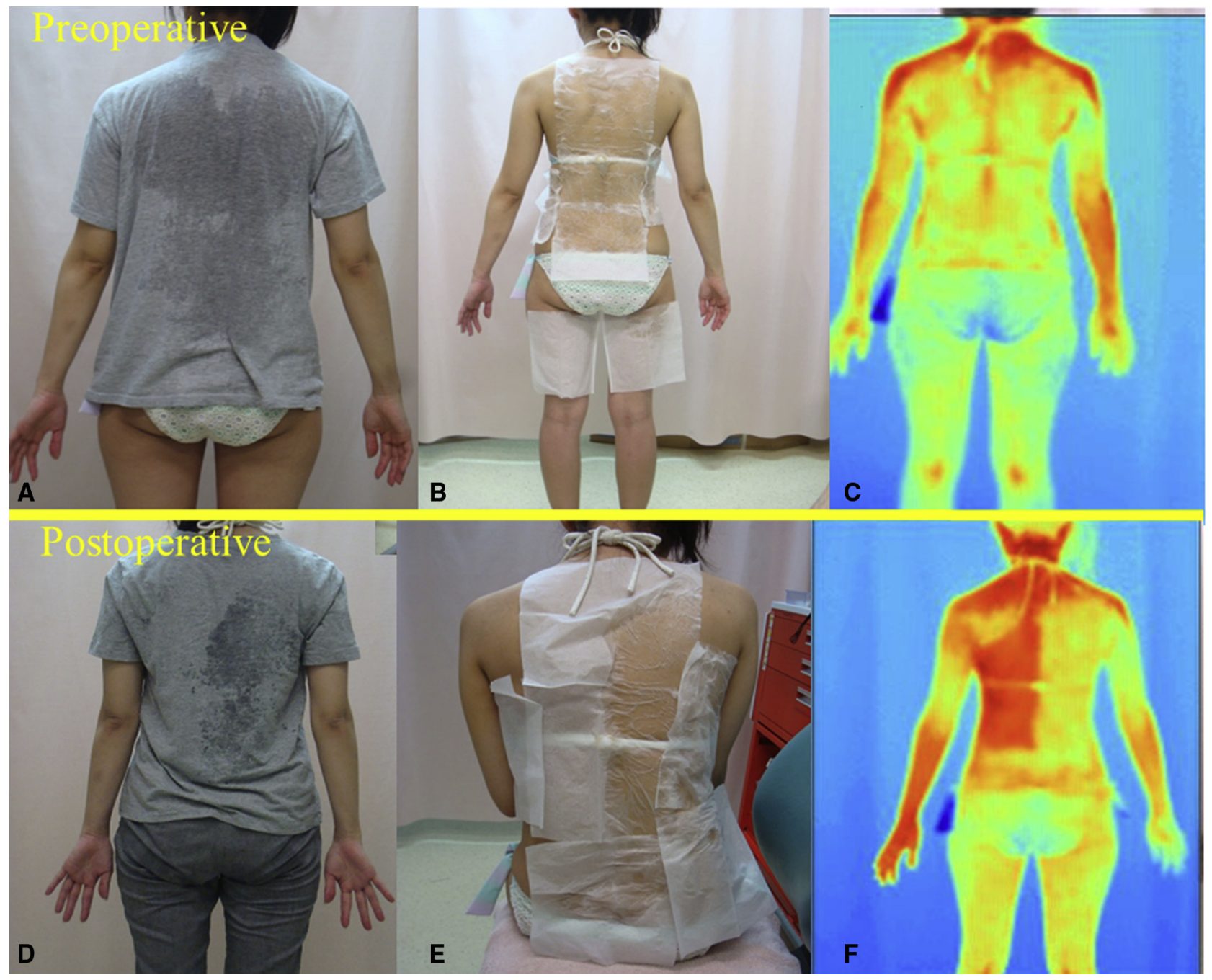

FIGURE 1. Sweat pattern and skin temperature of patient 1 after completion of 80 full squats. A, Typical compensatory sweating is recognized on the back. A clear boundary line between anhidrosis and hyperhidrosis is seen on the upper back. B, Kitchen paper sticks to the sweat from compensatory sweating after transection of the sympathetic trunk at the third and fourth ribs (see Panel A). C, Thermography for Panel A is shown. A low skin temperature is recorded in the area of compensatory sweating (see Panel A). D, Compensatory sweating of the left side has disappeared after ganglionectomy at the third, fourth, and fifth intercostal spaces. E, The sweating state of the back after ganglionectomy of the left side is shown. Sweating of the left side is decreased. F, Thermography of Panel $D$. The skin temperature on the left side has returned to normal. 

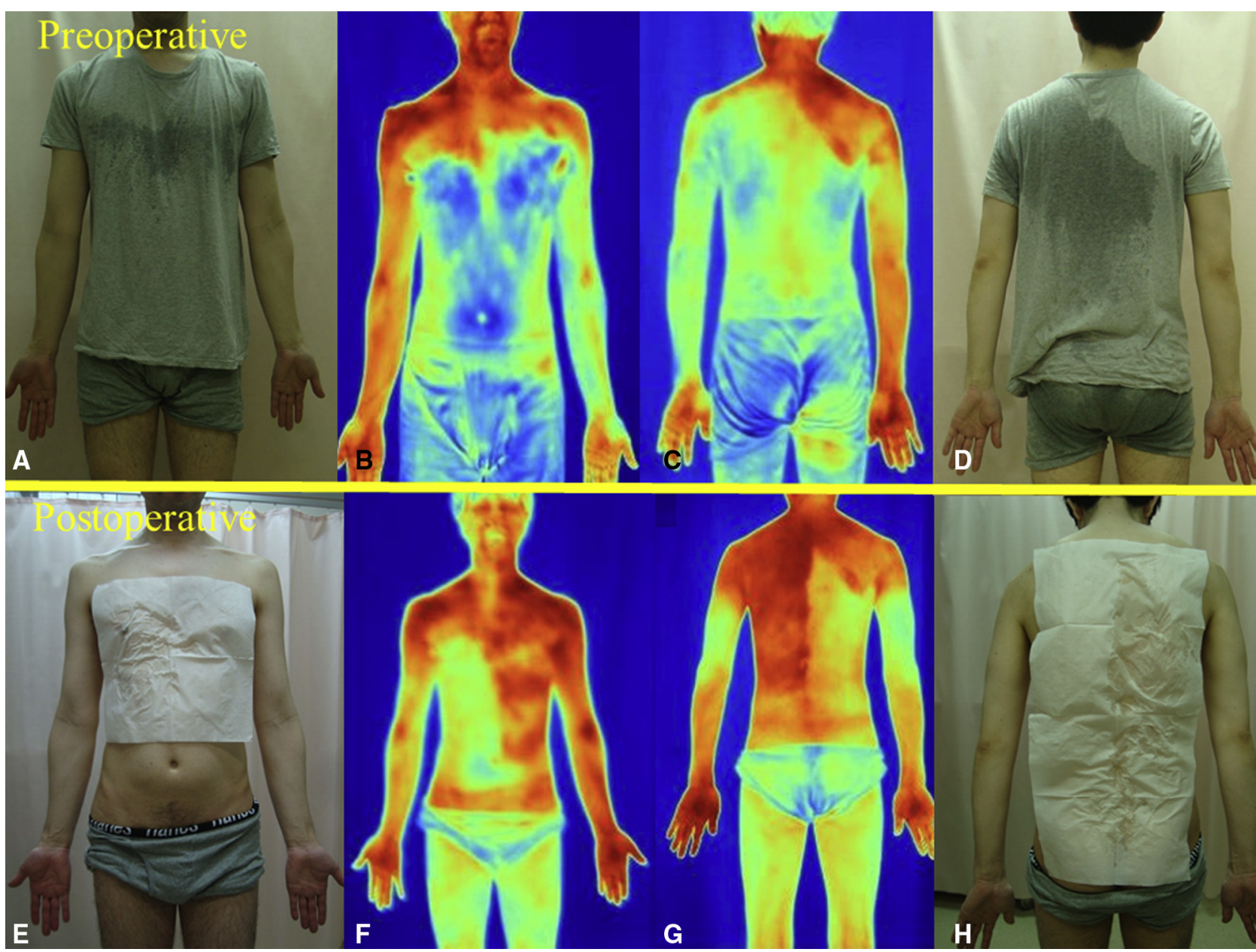

FIGURE 2. Postoperative sweat pattern and skin temperature after completion of 80 full squats (patient 5). A, Severe compensatory sweating is recognized on the chest, seen after bilateral thoracic sympathectomy was performed at the level of the third and fourth ribs; however, the boundary line of compensatory sweating varies in height between the sides. B, Thermography is shown after completion of 80 full squats. The low skin temperature area of the chest is larger than that on the right. C, Thermography shows that the low skin temperature area of the back is also larger than that on the right. D, The skin temperature reflects the state of compensatory sweating. E, The sweating state of the chest after ganglionectomy between the third rib and the eighth rib is shown. A significant decrease in compensatory sweating of the left chest is seen. F, The low skin temperature observed preoperatively on the left chest has resolved. G, The low skin temperature on the left back has also resolved. Ganglionectomy between the third rib and the eighth rib led to anhidrosis on the left side of the upper body. $\mathrm{H}$, The shape of the low temperature skin area indicates the state of sweating.

patient had undergone an initial sympathectomy, which involved transection of the sympathetic trunk, between July 1997 and August 2010. Palmar hyperhidrosis resolved in all cases; however, severe CS developed in all patients.

All patients received a detailed consultation to evaluate their personal, professional, and social handicaps related to CS. Preoperative chest computed tomography was performed to exclude lung or pleural disorders. Preoperative photography and thermography of CS were performed to estimate the change in CS (Figure 1, $A-C$; Figure 2, $A, B, E$, and $F$; and Figure 3, $A$ and $D$ ).

Patients were assessed for the amount and area of CS. In addition, a detailed medical history, including demographic data and clinical status, was documented. After careful explanation of the operative procedure, informed consent was obtained in writing at least 1 day before surgery.

\section{Surgical Procedure}

Ganglionectomy was performed in all patients unilaterally. On the basis of the evaluation of CS, each operation was performed with an interval of greater than 6 months, including the summer season. The procedure was performed with single-lung ventilation with the patient under general anesthesia and in the lateral decubitus position. The equipment included a thoracoscope $2 \mathrm{~mm}$ in diameter (2-mm MiniSite Gold Laparoscope; United States Surgical Corporation, Norwalk, Conn; or 2-mm K11605AA Miniature Straight Forward, Karl Storz SE \& Co. KG, Tuttlingen, Germany) and needle surgery devices consisting of 2 or 3 pieces.

A $3.0-\mathrm{mm}$ skin incision was made on the midaxillary line of the fourth, sixth, and seventh intercostal spaces. After temporary disconnection of the endotracheal tube, the thoracoscope was inserted into the pleural cavity. The sympathetic chain and ganglia were identified 


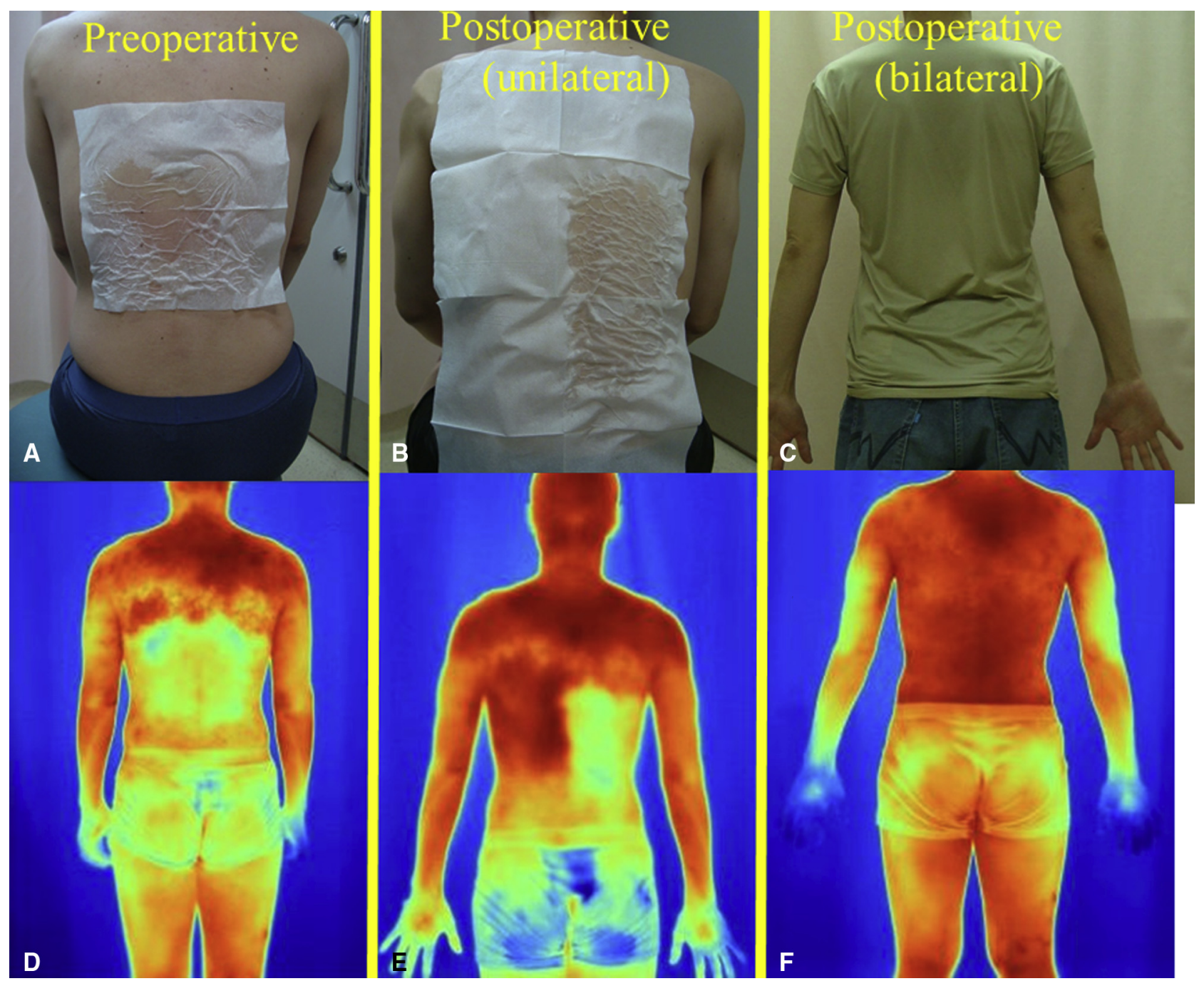

FIGURE 3. The postoperative sweat pattern and skin temperature (patient 4 ) after completion of 80 full squats. A, The sweating state of the back after thoracic sympathectomy was performed at the fourth and fifth ribs is shown. Typical compensatory sweating is seen on the back. B, The left ganglionectomy at the sixth, seventh, and eighth ribs has resulted in a significant decrease in the compensatory sweating of the left side after completion of 80 full squats. C, After the right ganglionectomy, performed similar to the one on the right side, compensatory sweating has resolved. No abnormal sweating is observed on the back. D, The skin temperature of the back after transection of the thoracic sympathetic trunk performed at the fourth and fifth ribs is shown. Typical compensatory sweating is accompanied by a low skin temperature. E, After the left ganglionectomy, the skin temperature of the left side has risen phenomenally. F, After the ganglionectomy on both sides, there is no area of low skin temperature across the whole back, even after completion of 80 full squats.

at the level of the second, third, fourth, and fifth costal heads by means of intraoperative chest $\mathrm{x}$-ray. When pneumopleural adhesions were found, all adhesions were divided at the location as near as possible to the lung.

Intraoperative monitoring with laser speckle flowgraphy (LSFG), as described previously and in ensuing text, ${ }^{7-9}$ was used to indicate the position of the CS. The ganglionectomy was then performed (Video 1).

After confirmation of the absence of bleeding and air leaks, the lung was inflated by the anesthetist. Air in the pleural space was expelled through the lumen of the scope sheath, and the scope itself was then removed. Sufficient expansion of the lung was confirmed with a second placement of the scope, before the scope and scope sheath were fully removed. Thoracotomy tubes were not used, and the wound was closed with sterile surgical tape. All patients were discharged on the operative day.

\section{Intraoperative Monitoring}

LSFG devices (LSFG-ANW; Soft Care Co Ltd, Fukutsu-shi, Japan; or OZ-2; Omegawave, Inc, Tokyo, Japan) were applied to identify the correct position of the sympathetic nerves and ganglia associated with CS. Transient changes in skin blood perfusion visible in the LSFG were monitored while stimulating each sympathetic nerve and ganglion with an electrosurgical unit (50-400V, 3-30 kHz, MS7000AS; Senko Medical Manufacturing Co Ltd, Tokyo, Japan). When a transient decrease in skin blood perfusion was observed with electrostimulation in the sympathetic nerves and ganglia, it was inferred that the target neural circuit related to CS was involved.

\section{Follow-up and Data Collection}

Follow-up was conducted during 3 visits at 3, 6, and 12 months after the operation to assess CS. Patients were required to fill out a detailed 


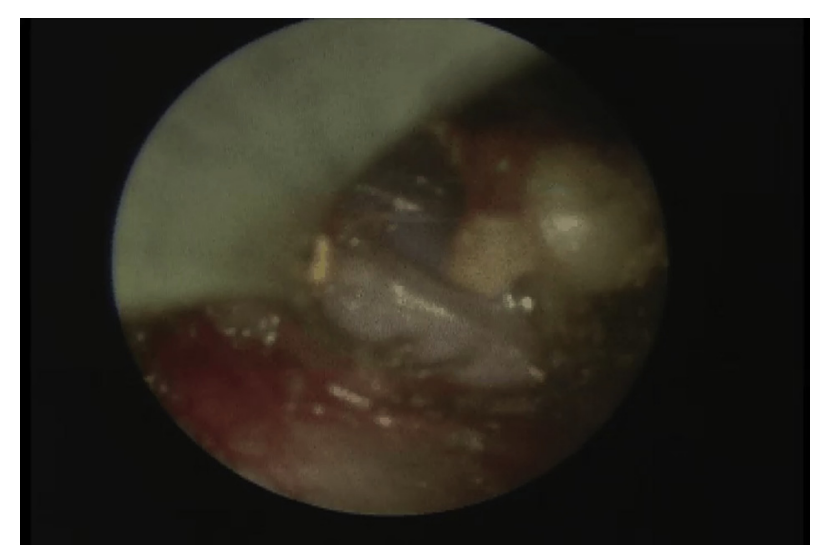

VIDEO 1. It is necessary to perform ganglionectomy to completely remove all of the sympathetic trunk and ganglia. Video available at: https://www.jtcvs.org/article/S0022-5223(19)31168-7/fulltext.

questionnaire. The data collected included resolution of symptoms, postoperative complications, severity of $\mathrm{CS}$, incidence of symptom recurrence, and satisfaction levels with the redo surgery. After the patient completed more than 2 sets of 40 full squats, the range of CS was evaluated with a photograph and thermograph in an environment with $55 \%$ humidity at room temperature $\left(29^{\circ} \mathrm{C}\right)$.

\section{RESULTS}

Eight patients were included in the study, and ganglionectomies were performed for each patient. The descriptive variables and operative details of each patient are summarized in Table 1 .

In all patients, a temporary decrease in skin blood perfusion was observed in the area of CS when stimulating sympathetic ganglia with LSFG (Figure 4, $A$ and $B$; Video 2). Ganglionectomy was performed at the same level as that of reaction the observed with LSFG. No conversions to open surgery were necessary, there were no deaths, and no intraoperative or postoperative complications, including Horner syndrome or pneumothorax, were observed. All patients were discharged on the operative day.

A summary of the patients' postoperative courses is shown in Table 1. The mean follow-up interval was $18.9 \pm 4.1$ (mean $\pm \mathrm{SD}$ ) months. Immediate amelioration of CS was noted after ganglionectomy, and the recurrence of CS was not reported during the follow-up in all patients. The satisfaction rate was $100 \%$.

\section{DISCUSSION}

For the treatment of CS, we first tried to conduct nerve interposition after ganglionectomy in several cases. ${ }^{10}$ In this series of CS treatments, we saw cases in which ganglionectomy alone successfully improved CS, although we failed to perform nerve interposition. There was no association between the interval of CS after the first ETS and the

TABLE 1. Descriptive variables and operative details of each patient

\begin{tabular}{|c|c|c|c|c|c|c|c|c|}
\hline Patient & 1 & 2 & 3 & 4 & 5 & 6 & 7 & 8 \\
\hline $\begin{array}{l}\text { Position of first } \\
\text { sympathectomy }\end{array}$ & $\mathrm{T} 3+\mathrm{T} 4$ & $\mathrm{~T} 3+\mathrm{T} 4$ & $\mathrm{~T} 3+\mathrm{T} 4$ & $\mathrm{~T} 4+\mathrm{T} 5$ & $\mathrm{~T} 3+\mathrm{T} 4+\mathrm{T} 5$ & $\mathrm{~T} 3+\mathrm{T} 4+\mathrm{T} 5$ & $\mathrm{~T} 2+\mathrm{T} 3+\mathrm{T} 4$ & $\mathrm{~T} 2+\mathrm{T} 3$ \\
\hline Age (y) & 27 & 26 & 24 & 33 & 32 & 54 & 36 & 46 \\
\hline Sex & $\mathrm{F}$ & M & M & M & M & M & M & M \\
\hline $\begin{array}{l}\text { Preoperative } \\
\text { severity of CS }\end{array}$ & $\mathrm{Se}$ & $\mathrm{Se}$ & $\mathrm{Se}$ & $\mathrm{Se}$ & $\mathrm{Se}$ & $\mathrm{Se}$ & $\mathrm{Se}$ & $\mathrm{Se}$ \\
\hline Operation side & Both & Both & Right & Both & Both & Both & Both & Both \\
\hline $\begin{array}{l}\text { Levels of } \\
\text { ganglionectomy } \\
\text { for CS }\end{array}$ & $\begin{array}{c}\mathrm{T} 3+\mathrm{T} 4+ \\
\mathrm{T} 5\end{array}$ & $\begin{array}{c}\mathrm{T} 3+\mathrm{T} 4+ \\
\mathrm{T} 5+\mathrm{T} 6+ \\
\mathrm{T} 7\end{array}$ & $\begin{array}{c}\mathrm{T} 5+\mathrm{T} 6+ \\
\mathrm{T} 7\end{array}$ & $\begin{array}{c}\mathrm{T} 6+\mathrm{T} 7+ \\
\mathrm{T} 8\end{array}$ & $\begin{array}{c}\mathrm{T} 3+\mathrm{T} 4+ \\
\mathrm{T} 5+\mathrm{T} 6+ \\
\mathrm{T} 7\end{array}$ & $\begin{array}{c}\mathrm{T} 3+\mathrm{T} 4+ \\
\mathrm{T} 5+\mathrm{T} 6+ \\
\mathrm{T} 7\end{array}$ & $\begin{array}{r}\mathrm{T} 3+\mathrm{T} 4+ \\
\mathrm{T} 5+\mathrm{T} 6\end{array}$ & $\begin{array}{c}\mathrm{T} 3+\mathrm{T} 4+ \\
\mathrm{T} 5\end{array}$ \\
\hline Duration (min) & 173 & 160 & 125 & 225 & 174 & 186 & 178 & 182 \\
\hline Adhesion & + & + & + & + & + & + & - & + \\
\hline Follow-up (mo) & 12 & 13 & 16 & 19 & 20 & 22 & 23 & 26 \\
\hline $\begin{array}{l}\text { Severity of CS } \\
12 \text { mo after } \\
\text { ganglionectomy }\end{array}$ & $\mathrm{N}$ & $\mathrm{N}$ & $\mathrm{L}$ & $\mathrm{N}$ & $\mathrm{N}$ & $\mathrm{L}$ & $\mathrm{N}$ & $\mathrm{L}$ \\
\hline $\begin{array}{l}\text { Satisfaction levels } \\
12 \text { mo after } \\
\text { ganglionectomy }\end{array}$ & VS & VS & VS & VS & VS & VS & VS & VS \\
\hline
\end{tabular}

Transection levels: $T 2$, Second rib; $T 3$, third rib; $T 4$, fourth rib; $T 5$, fifth rib; $T 6$, sixth rib; $T 7$, seventh rib. Severity levels (decreasing order): $S e$, Severe; $M o$, moderate; $M i$, mild; $L$, little; $N$, none. Patient satisfaction levels: $V S$, very satisfied; $S$, satisfied; $D$, dissatisfied; $V D$, very dissatisfied; $C S$, Compensatory sweating. 

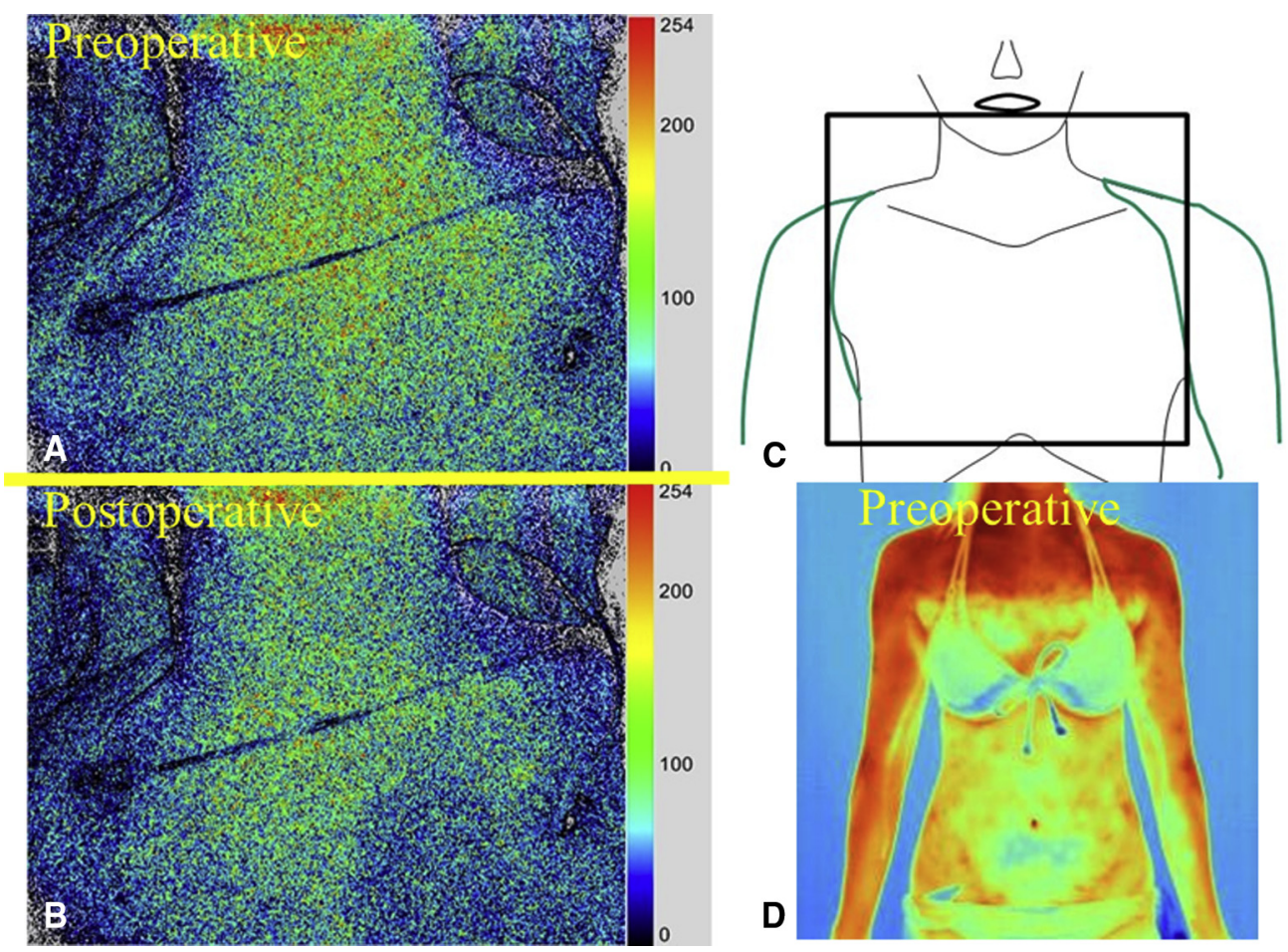

FIGURE 4. Intraoperative laser speckle flowgraphy images of the precordial region (patient 1). A, An image before electrical stimulation of the thoracic sympathetic trunk is shown. The colors indicate skin blood perfusion, with the vertical color bar reflecting the standard. B, An image of the precordial region after electrical stimulation of the left fourth thoracic sympathetic ganglion. A decrease in the skin blood perfusion of the left subclavian area is observed. The ganglion is therefore considered to be related to the level of skin perfusion. C, Examination area of the laser speckle flowgraphy is shown. D, Skin temperature of patient 1 after completion of 80 full squats. A low skin temperature of the anterior chest is recorded in the area of compensatory sweating (see Figure 1, A-C).

results of the ganglionectomy. This finding encouraged us to investigate the effectiveness of ganglionectomy against CS. It is thought that sudomotor nerves related to CS are involved in the ganglia.

In the skin area where CS was improved by ganglionectomy, a phenomenal rise in the skin temperature could be observed by a thermograph postoperatively. Blood perfusion to the skin was thus increased. We therefore attempted to observe the skin temperature intraoperatively; however, it was very difficult to find a significant change, because of the slow speed of temperature change. We therefore decided to measure the skin blood flow directly with LSFG, because the change in LSFG occurs within several seconds after stimulation of the ganglia for 0.1 second.

We recorded CS under 5 categories (Se, severe; Mo, moderate; $\mathrm{Mi}$, mild; $\mathrm{L}$, little; and $\mathrm{N}$, none). Figure 5 shows the flowchart of patient selection. Around 30\% of patients answered none, but $385(3 \%)$ answered severe. The results of the severity of CS changed according to the excision method. All patients $(n=13,089)$ were told of the possibility of severe CS before thoracic sympathectomy. All patients recognized that there was little scope to improve the severity of CS. In addition, we do not emphasize the possibility of treatment with this technique, because there is the possibility that the operation will not go as planned if there are severe pneumopleural adhesions. As a result, few patients were considered candidates for ganglionectomy with LSFG with the intent of treating CS (Figure 5).

We rarely observed bradycardia or hypotension in this series, because we did not treat ganglionectomy between the second and third ribs. We have been performing ganglionectomy for more than 10 years for patients with hyperhidrosis. So far, there have not been any long-term cardiac issues. Adequate selection of ganglionectomies for a patient with local hyperhidrosis decreases the postoperative complaints, including coldness of the hand, CS, and other indefinite complaints.

We routinely perform ganglionectomy for new patients with hyperhidrosis. Ganglionectomy of the appropriate positions decrease the incidence of various postoperative problems.

These patients underwent the first ETS on one side. Because they did not have severe CS after the first operation, they then underwent ETS on the opposite side. 


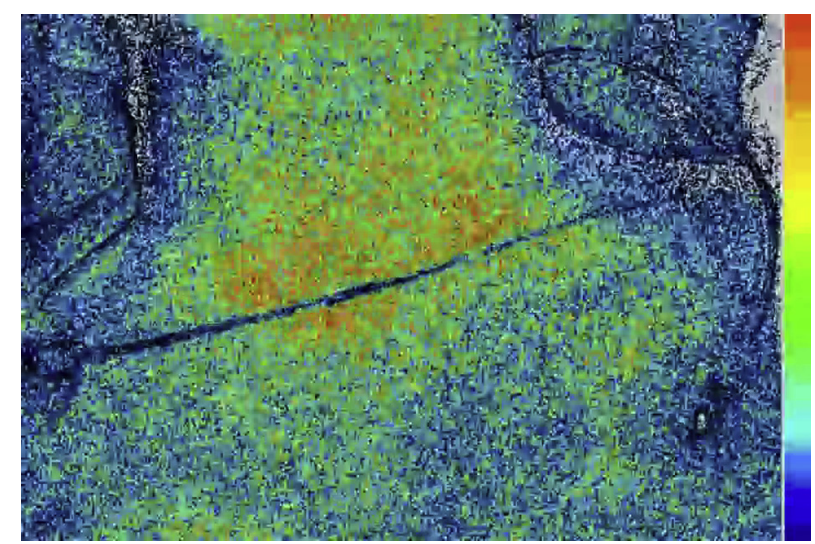

VIDEO 2. Skin blood perfusion $(S B P)$ is shown by a color change. Vertical color bar is the standard. A temporary decrease of skin blood perfusion in the left subclavian area was observed immediately after electrical stimulation at the fifth, fourth, and third sympathetic ganglia. Video available at: https://www.jtcvs.org/article/S0022-5223(19)31168-7/fulltext.

There are many patients who undergo their first operation on the right side. Because there was significant anamnesis, we began often to perform the left-sided treatment first. We believe that there would be no difference in undertaking the operation from either side. In this series, 7 patients who underwent previous ETS of both sides advanced to ganglionectomy with this LSFG technique; the interval between the 2 procedures was greater than 6 months.

LSFG devices can be used to evaluate skin blood perfusion noninvasively. The laser speckle phenomenon is an interference event observed when coherent light sources, such as lasers, are scattered by a diffusing surface. The speckle pattern appears in accordance with the blood flow velocity. $^{7-9}$ There are 2 companies that manufacture LSFG in Japan, so LSFG is readily available here. Ophthalmologists examining the retina often use LSFG clinically. The cost of LSFG is about $\$ 30,000.00$ US. We did not charge the patients for the technology in the operating room.

We applied the LSFG technique to treat CS in a targeted manner. A change in LSFG could be repeatedly observed when electrically stimulating each sympathetic nerve (Figure 4, B; and Video 2). On the other hand, the device is susceptible to overheating. When an energy device is used for electrical stimulation, it is necessary to be careful not to overheat the ganglion. The electrical stimulation starts with low power first and gradually increases. The technique was successful in most cases in this series. The skin area where the LSFG reaction is identified by electric stimulation allows complete removal of the sympathetic neuron, as in a ganglionectomy. Insufficient ablation and clip application are factors associated with the recurrence of CS.
Local hyperhidrosis is thought to be caused by increased sympathetic signaling to the sweat glands of the palm and axillary region. ${ }^{11}$ ETS interrupts this signaling. There is vast evidence to indicate that there is a nerve system emitting a large quantity of sudomotor signaling to the skin where CS appears. Interrupting one of the nerve circuits related to CS could result in CS being ameliorated; however, it has proved difficult to detect precisely where the nerve circuitry is located.

After ETS, skin temperature was increased in the skin domain where sweating was decreased because of the quantity of blood circulating in the skin. Two functions of the sympathetic nerves, vasomotor action and sudomotor action, have been known to act in the same skin domain.

Sudomotor action is reduced by general anesthesia, but vasomotor action can still function. Skin blood perfusion can be observed by application of LSFG with the patient under general anesthesia. This is the reason for using LSFG. CS was decreased by dividing the sympathetic nerve related to the CS; adequate excision of the sympathetic nerve responsible for CS can therefore be used to treat CS.

CS after ETS has been previously explained as a thermoregulatory response to the anhidrosis induced by ETS. ${ }^{3-6}$ It is doubtful, however, that the large quantity of sweat observed in CS aids in body temperature control. Actually, we showed that CS did not increase on the other surfaces, although the area of anhidrosis was extended. Patients did not lose thermoregulatory function after the ganglionectomy because the emission of heat radiation increased from the anhidrosis area. The body temperature of the patients was thus kept constant during exercise. CS is therefore not thought to be brought about by a thermoregulatory response. All patients were almost completely satisfied with the results, because their CS was decreased.

Other kinds of hyperhidrosis, such as on the back, chest, and stomach, are also treatable with this technique. LSFG could be applied for the treatment of facial blushing ${ }^{12}$ or ischemic disease, such as Buerger disease and Raynaud disease.

In conclusion, with the LSFG technique, sympathetic nerves related to the excessive sweating of various body parts could be identified, resulting in the sweating being controlled. Indications for ETS have been restricted because of the side effect of CS; however, if the problem of CS is resolved, these indications would be further extended.

\section{Conflict of Interest Statement}

Authors have nothing to disclose with regard to commercial support. 


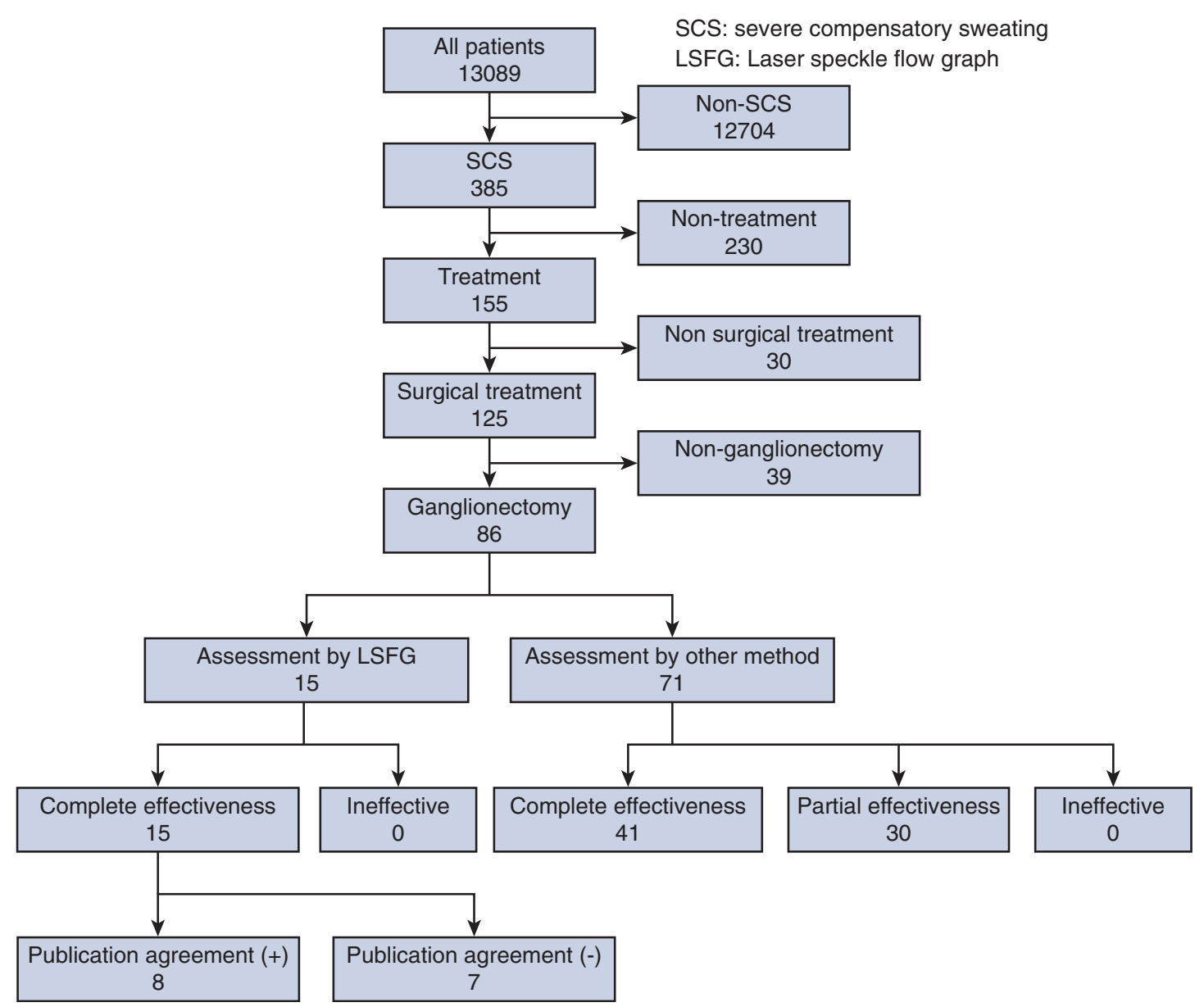

FIGURE 5. Flowchart of patient selection. We recorded compensatory sweating under 5 categories ( $\mathrm{Se}$, severe; $\mathrm{Mo}$, moderate; $M i$, mild; $L$, little; $N$, none). Around $30 \%$ of all patients answered none, but $385(3 \%)$ answered severe. The results of the severity of compensatory sweating changed according to the excision method. All patients $(n=13,089)$ were told of the possibility of severe compensatory sweating before thoracic sympathectomy. All patients recognized that there was little scope to improve the severity of compensatory sweating; however, 155 patients underwent surgical treatment. In 30 patients, the operation did not go as planned because of severe pneumopleural adhesions. Among the 125 patients undergoing surgical treatment, 86 patients underwent ganglionectomy. The skin area with anhidrosis was always confirmed in the patients with compensatory sweating who underwent ganglionectomy. Intraoperative laser speckle flowgraphy $(L S F G)$ was effective as a technique to partially control the anhidrosis. SCS, Severe compensatory sweating.

\section{References}

1. Strutton DR, Kowalski JW, Glaser DA, Stang PE. US prevalence of hyperhidrosis and impact on individuals with axillary hyperhidrosis: results from a national survey. J Am Acad Dermatol. 2004;51:241-8.

2. Fujimoto T, Kawahara K, Yokozeki H. Epidemiological study and considerations of primary focal hyperhidrosis in Japan: from questionnaire analysis. J Dermatol. 2013;40:886-90.

3. Shelley WB, Florence R. Compensatory hyperhidrosis after sympathectomy. $N$ Engl J Med. 1960;263:1056-8.

4. Andrews BT, Rennie JA. Predicting changes in the distribution of sweating following thoracoscopic sympathectomy. Br J Surg. 1997;84:1702-4.

5. Lesèche G, Castier Y, Thabut G, Petit MD, Combes M, Cerceau O, et al. Endoscopic transthoracic sympathectomy for upper limb hyperhidrosis: limited sympathectomy does not reduce postoperative compensatory sweating. J Vasc Surg. 2003;37:124-8.

6. Gossot D, Toledo L, Fritsch S, Célérier M. Thoracoscopic sympathectomy for upper limb hyperhidrosis: looking for the right operation. Ann Thorac Surg. 1997;64:975-8.
7. Lee MC, Konishi N, Fujii H. Blood flow analyses of skin tissue under the sacrum using laser speckle flowgraphy. Opt Rev. 2003;10:562-6.

8. Yamamoto $H$. The observation of body surface circulatory dynamics using LASER Speckle Flow Meter in the thoracic sympathectomy. Jpn J Laser Surg Med. 2014;35:180-6.

9. Sugiyama T, Araie M, Riva CE, Schmetterer L, Orgul S. Use of laser speckle flowgraphy in ocular blood flow research. Acta Ophthalmol. 2010;88:723-9.

10. Telaranta T. Reversal surgery for reducing the side effects of ETS. A case report. Ann Chir Gynaecol. 2001;90:175-6.

11. Ray BS, Hinsey JC, Geohegan WA. Observations on the distribution of the sympathetic nerves to pupil and upper extremity as determined by stimulation of the anterior roots in man. Ann Surg. 1943;118:647-55.

12. Yamamoto H, Okada M. Sympathetic ganglionectomy for facial blushing using application of laser speckle flow graph. J Thorac Cardiovasc Surg. 2018;156:1326-31.

Key Words: compensatory sweating, ganglionectomy, laser speckle flowgraphy 